Kragujevac Journal of Mathematics

Volume 44(4) (2020), Pages 563-570.

\title{
A NOTE ON THE DEFINITION OF BOUNDED VARIATION OF HIGHER ORDER FOR DOUBLE SEQUENCES
}

\author{
BHIKHA LILA GHODADRA ${ }^{1}$ AND VANDA FÜLÖP ${ }^{2}$
}

\begin{abstract}
In this study the definition of bounded variation of order $p(p \in \mathbb{N})$ for double sequences is considered. Some inclusion relations are proved and counter examples are provided for ensuring proper inclusions.
\end{abstract}

\section{INTRODUCTION}

While studying convergence properties of double trigonometric and Walsh series, many authors have considered double sequences which are of bounded variation or more generally of bounded variation of order $(p, 0),(0, p)$, and $(p, p)$ (see, e.g., $[1$, $3]$ ). Also, many results regarding the convergence of trigonometric and Walsh series with coefficients of bounded variation of higher order are proved (see, e.g., [1,3]). But, it seems that showing the inclusion relations for such classes of sequences and constructing counter examples for showing proper inclusions have not yet been done.

The main goal of this note is to prove such inclusion relations and constructing examples for showing proper inclusions. We start with the one dimensional case. In what follows, by a sequence (or a single sequence), we mean a function from $\mathbb{Z}$ to $\mathbb{C}$, and by a double sequence, we mean a function from $\mathbb{Z} \times \mathbb{Z}$ to $\mathbb{C}$.

\section{One Dimensional Case}

We recall the definition of bounded variation of order $p$ for a single sequence (see [2, Defintion 1.4]).

Key words and phrases. Double sequence of bounded variation, double sequence of bounded variation of order $p(p \in \mathbb{N})$, double sequence of bounded variation of order $(p, 0)$, double sequence of bounded variation of order $(0, p)$, double sequence of bounded variation of order $(p, p)$.

2010 Mathematics Subject Classification. Primary: 40B05.

DOI $10.46793 / \mathrm{KgJMat} 2004.563 \mathrm{G}$

Received: April 20, 2018.

Accepted: July 02, 2018. 
Definition 2.1. A null sequence $\left\{a_{k}: k=\ldots,-1,0,1, \ldots\right\}$, i.e., $\left\{a_{k}\right\}$ such that $a_{k} \rightarrow 0$ as $|k| \rightarrow \infty$, is said to be of bounded variation of order $p(p \in \mathbb{N})$ if

$$
\sum_{k=-\infty}^{\infty}\left|\Delta^{p} a_{k}\right|<\infty
$$

where $\Delta^{p} a_{k}=\Delta\left(\Delta^{p-1} a_{k}\right)=\Delta^{p-1} a_{k}-\Delta^{p-1} a_{k+1}$ and $\Delta^{0} a_{k}=a_{k}$.

From this definition, it is clear that if $\left\{a_{k}\right\}$ is of bounded variation of order $p$, then it is of bounded variation of order $p+1$ also. Also, in [2] an example of a sequence is given which is of bounded variation of order 2, but not of bounded variation.

\section{Two Dimensional Case}

In this section, we shall consider the definition of a double sequence of bounded variation of order $p$. For that first we have the following definition of differences.

Definition 3.1. Let $\{c(j, k): j, k=\ldots,-1,0,1, \ldots\}$ be a double sequence. Its differences are defined by

$$
\begin{aligned}
& \Delta_{00} c(j, k)=c(j, k) \\
& \Delta_{p q} c(j, k)=\Delta_{p-1, q} c(j, k)-\Delta_{p-1, q} c(j+1, k), \quad p \geq 1, \\
& \Delta_{p q} c(j, k)=\Delta_{p, q-1} c(j, k)-\Delta_{p, q-1} c(j, k+1), \quad q \geq 1 .
\end{aligned}
$$

As is well-known, the two right-hand sides coincide if $\min (p, q) \geq 1$. Also, we mention that that double induction argument gives

$$
\Delta_{p q} c(j, k)=\sum_{s=0}^{p} \sum_{t=0}^{q}(-1)^{s+t}\left(\begin{array}{l}
p \\
s
\end{array}\right)\left(\begin{array}{l}
q \\
t
\end{array}\right) c(j+s, k+t) .
$$

Definition 3.2. A double sequence $\{c(j, k): j, k=\ldots,-1,0,1, \ldots\}$ of complex numbers is called a null sequence, if it satisfies

$$
c(j, k) \rightarrow 0 \quad \text { as } \max (|j|,|k|) \rightarrow \infty .
$$

Definition 3.3. A double null sequence $\{c(j, k): j, k=\ldots,-1,0,1, \ldots\}$ is said to be of bounded variation if

$$
\sum_{j=-\infty}^{\infty} \sum_{k=-\infty}^{\infty}\left|\Delta_{11} c(j, k)\right|<\infty
$$

We shall denote the class of all double sequences of bounded variation by $\mathfrak{B V}$.

Now, we give an analogous definition of bounded variation of order $p(p \geq 2)$ for a double sequence.

Definition 3.4. A double null sequence $\{c(j, k): j, k=\ldots,-1,0,1, \ldots\}$ is said to belong to the class $(\mathfrak{B V V})^{p}$, i.e., of bounded variation of order $p \geq 2$, if the following three conditions are satisfied:

$$
\sum_{j=-\infty}^{\infty} \sum_{k=-\infty}^{\infty}\left|\Delta_{p p} c(j, k)\right|<\infty
$$




$$
\lim _{|k| \rightarrow \infty} \sum_{j=-\infty}^{\infty}\left|\Delta_{p 0} c(j, k)\right|=0
$$

and

$$
\lim _{|j| \rightarrow \infty} \sum_{k=-\infty}^{\infty}\left|\Delta_{0 p} c(j, k)\right|=0 .
$$

Some authors (see, e.g., [1,3]) call conditions (3.3)-(3.5) as conditions of bounded variation of order $(p, p),(p, 0)$, and $(0, p)$, respectively.

Our main aim is to prove that the following chain of inclusion relations holds:

$$
\mathfrak{B V}=(\mathfrak{B V})^{1} \subset(\mathfrak{B V})^{2} \subset \cdots \subset(\mathfrak{B V})^{p} \subset(\mathfrak{B V})^{p+1} \subset \cdots .
$$

We also show that each of above relation is proper.

Theorem 3.1. If $\{c(j, k)\} \in \mathfrak{B V}$, then $\{c(j, k)\} \in(\mathfrak{B V})^{2}$.

Proof. Suppose $\{c(j, k)\} \in \mathfrak{B V}$. Then, we write

$$
\sum_{j=-\infty}^{\infty} \sum_{k=-\infty}^{\infty}\left|\Delta_{11} c(j, k)\right|<\infty .
$$

Since

$$
\begin{aligned}
\Delta_{22} c(j, k) & =\Delta_{12} c(j, k)-\Delta_{12} c(j+1, k) \\
& =\Delta_{11} c(j, k)-\Delta_{11} c(j, k+1)-\Delta_{11} c(j+1, k)+\Delta_{11} c(j+1, k+1),
\end{aligned}
$$

it follows from (3.6) that

$$
\sum_{j=-\infty}^{\infty} \sum_{k=-\infty}^{\infty}\left|\Delta_{22} c(j, k)\right| \leq 4 \sum_{j=-\infty}^{\infty} \sum_{k=-\infty}^{\infty}\left|\Delta_{11} c(j, k)\right|<\infty .
$$

So, $\{c(j, k)\}$ satisfies (3.3) for $p=2$. Now, as $\{c(j, k)\}$ is of bounded variation, that is, $\{c(j, k)\}$ is a double sequence satisfying (3.1) and (3.2), it follows (see, e.g., [4, Proof of Lemma 1]) that

$$
\Delta_{10} c\left(j, k_{0}\right)=\sum_{k=k_{0}}^{\infty} \Delta_{11} c(j, k) \text { and } \Delta_{10} c\left(j, k_{0}\right)=-\sum_{k=-\infty}^{k_{0}-1} \Delta_{11} c(j, k),
$$

for each fixed $k_{0} \in \mathbb{Z}$. Therefore, for each fixed $k_{0} \in \mathbb{Z}$, we have

$$
\begin{aligned}
\left|\Delta_{20} c\left(j, k_{0}\right)\right| & =\left|\Delta_{10} c\left(j, k_{0}\right)-\Delta_{10} c\left(j+1, k_{0}\right)\right| \\
& =\left|\sum_{k=k_{0}}^{\infty} \Delta_{11} c(j, k)-\sum_{k=k_{0}}^{\infty} \Delta_{11} c(j+1, k)\right| \\
& \leq \sum_{k=k_{0}}^{\infty}\left|\Delta_{11} c(j, k)\right|+\sum_{k=k_{0}}^{\infty}\left|\Delta_{11} c(j+1, k)\right|
\end{aligned}
$$

and

$$
\left|\Delta_{20} c\left(j, k_{0}\right)\right|=\left|\Delta_{10} c\left(j, k_{0}\right)-\Delta_{10} c\left(j+1, k_{0}\right)\right|
$$




$$
\begin{aligned}
& =\left|-\sum_{k=-\infty}^{k_{0}-1} \Delta_{11} c(j, k)+\sum_{k=-\infty}^{k_{0}-1} \Delta_{11} c(j+1, k)\right| \\
& \leq \sum_{k=-\infty}^{k_{0}-1}\left|\Delta_{11} c(j, k)\right|+\sum_{k=-\infty}^{k_{0}-1}\left|\Delta_{11} c(j+1, k)\right| .
\end{aligned}
$$

Therefore, in view of (3.6), we have

$$
\begin{aligned}
\sum_{j=-\infty}^{\infty}\left|\Delta_{20} c\left(j, k_{0}\right)\right| & \leq \sum_{j=-\infty}^{\infty} \sum_{k=k_{0}}^{\infty}\left|\Delta_{11} c(j, k)\right|+\sum_{j=-\infty}^{\infty} \sum_{k=k_{0}}^{\infty}\left|\Delta_{11} c(j+1, k)\right| \\
& \rightarrow 0 \text { as } k_{0} \rightarrow \infty
\end{aligned}
$$

and

$$
\begin{aligned}
\sum_{j=-\infty}^{\infty}\left|\Delta_{20} c\left(j, k_{0}\right)\right| & \leq \sum_{j=-\infty}^{\infty} \sum_{k=-\infty}^{k_{0}-1}\left|\Delta_{11} c(j, k)\right|+\sum_{j=-\infty}^{\infty} \sum_{k=-\infty}^{k_{0}-1}\left|\Delta_{11} c(j+1, k)\right| \\
& \rightarrow 0 \text { as } k_{0} \rightarrow-\infty
\end{aligned}
$$

Therefore, $\{c(j, k)\}$ satisfies (3.4) for $p=2$. Similarly, it satisfies (3.5) for $p=2$. Thus, $\{c(j, k)\} \in(\mathfrak{B V})^{2}$. This completes the proof.

Theorem 3.2. If $\{c(j, k)\} \in(\mathfrak{B V})^{p}, p \geq 2$, then $\{c(j, k)\} \in(\mathfrak{B V})^{p+1}$.

Proof. Suppose $\{c(j, k)\} \in(\mathfrak{B V})^{p}$. Then, (3.3)-(3.5) hold true. Since

$$
\begin{aligned}
\Delta_{p+1, p+1} c(j, k) & =\Delta_{p, p+1} c(j, k)-\Delta_{p, p+1} c(j+1, k) \\
& =\Delta_{p p} c(j, k)-\Delta_{p p} c(j, k+1)-\Delta_{p p} c(j+1, k)+\Delta_{p p} c(j+1, k+1),
\end{aligned}
$$

it follows from (3.3) that

$$
\sum_{j=-\infty}^{\infty} \sum_{k=-\infty}^{\infty}\left|\Delta_{p+1, p+1} c(j, k)\right| \leq 4 \sum_{j=-\infty}^{\infty} \sum_{k=-\infty}^{\infty}\left|\Delta_{p p} c(j, k)\right|<\infty .
$$

So, $\{c(j, k)\}$ satisfies $(3.3)$ for $p+1$ in place of $p$. Also, for a fixed $k \in \mathbb{Z}$, we have

$$
\begin{aligned}
\sum_{j=-\infty}^{\infty}\left|\Delta_{p+1,0} c(j, k)\right| & =\sum_{j=-\infty}^{\infty}\left|\Delta_{p, 0} c(j, k)-\Delta_{p, 0} c(j+1, k)\right| \\
& \leq \sum_{j=-\infty}^{\infty}\left|\Delta_{p, 0} c(j, k)\right|+\sum_{j=-\infty}^{\infty}\left|\Delta_{p, 0} c(j+1, k)\right| \\
& \rightarrow 0 \text { as }|k| \rightarrow \infty
\end{aligned}
$$

in view of (3.4). So, $\{c(j, k)\}$ satisfies (3.4) for $p+1$ in place of $p$. Similarly, in view of (3.5), $\{c(j, k)\}$ satisfies (3.5) for $p+1$ in place of $p$. Therefore, $\{c(j, k)\}$ is of bounded variation of order $p+1$.

Now, we will prove that the inclusion relations proved in above theorems are proper. In the following example, we give an example of a double sequence defined on $\mathbb{Z} \times \mathbb{Z}$, which is of bounded variation of of order 2 , but not of bounded variation. 
Example 3.1. We consider $\left\{a_{j}\right\}$ and $\left\{b_{k}\right\}$ to be single sequences as in [2, Example, p. 424]. That is, for $j, k=1,2, \ldots$, and $-j \leq p<j,-k \leq q<k$, we put

$$
a_{j^{2}+p}=\frac{j-|p|}{j^{2}}, \quad b_{k^{2}+q}=\frac{k-|q|}{k^{2}} .
$$

As argued in [2], the sequences $\left\{a_{j}\right\}$ and $\left\{b_{k}\right\}$ are well-defined on $\mathbb{N} \cup\{0\}$ as $j^{2}+j=$ $(j+1)^{2}-(j+1)$ and $k^{2}+k=(k+1)^{2}-(k+1)$. We also put $a_{j}=0$ if $j \leq-1$, and $b_{k}=0$ if $k \leq-1$. Then, $\left\{a_{j}\right\}$ and $\left\{b_{k}\right\}$ are well-defined sequences on $\mathbb{Z}$.

Now, we put

$$
c(j, k)=a_{j} b_{k}, \quad j, k \in \mathbb{Z} .
$$

Then, $\{c(j, k)\}$ is a well-defined double sequence on $\mathbb{Z} \times \mathbb{Z}$. Actually, it is proved in [2] that these single sequences are of bounded variation of order 2 , but not of bounded variation. We claim that the double sequence $\{c(j, k)\}$ is of bounded variation of order 2 , but not of bounded variation.

We first observe that

$$
\begin{aligned}
\Delta_{11} c(j, k) & =c(j, k)-c(j+1, k)-c(j, k+1)+c(j+1, k+1) \\
& =a_{j} b_{k}-a_{j+1} b_{k}-a_{j} b_{k+1}+a_{j+1} b_{k+1} \\
& =\left(a_{j}-a_{j+1}\right) b_{k}-\left(a_{j}-a_{j+1}\right) b_{k+1} \\
& =\left(\Delta a_{j}\right) b_{k}-\left(\Delta a_{j}\right) b_{k+1} \\
& =\left(\Delta a_{j}\right)\left(\Delta b_{k}\right)
\end{aligned}
$$

and therefore, we also have

$$
\begin{aligned}
\Delta_{22} c(j, k) & =\Delta_{12} c(j, k)-\Delta_{12} c(j+1, k) \\
& =\Delta_{11} c(j, k)-\Delta_{11} c(j, k+1)-\Delta_{11} c(j+1, k)+\Delta_{11} c(j+1, k+1) \\
& =\left(\Delta a_{j}\right)\left(\Delta b_{k}\right)-\left(\Delta a_{j}\right)\left(\Delta b_{k+1}\right)-\left(\Delta a_{j+1}\right)\left(\Delta b_{k}\right)+\left(\Delta a_{j+1}\right)\left(\Delta b_{k+1}\right) \\
& =\left(\Delta a_{j}\right)\left(\Delta b_{k}-\Delta b_{k+1}\right)-\left(\Delta a_{j+1}\right)\left(\Delta b_{k}-\Delta b_{k+1}\right) \\
& =\left(\Delta^{2} a_{j}\right)\left(\Delta^{2} b_{k}\right) .
\end{aligned}
$$

Now, by definition of $\left\{a_{j}\right\}$,

$$
\begin{aligned}
\Delta a_{j^{2}+p} & =a_{j^{2}+p}-a_{j^{2}+p+1}=\frac{j-|p|}{j^{2}}-\frac{j-|p+1|}{j^{2}}=\frac{|p+1|-|p|}{j^{2}} \\
& = \begin{cases}\frac{1}{j^{2}}, & \text { if } p \geq 0, \\
\frac{-1}{j^{2}}, & \text { if } p \leq-1 .\end{cases}
\end{aligned}
$$

Therefore, $\left|\Delta a_{j^{2}+p}\right|=1 / j^{2}$ and similarly $\left|\Delta b_{k^{2}+q}\right|=1 / k^{2}$. Next, we have

$$
\sum_{j=0}^{\infty}\left|\Delta a_{j}\right|=\sum_{j=1}^{\infty} \sum_{p=-j}^{j-1}\left|\Delta a_{j^{2}+p}\right|=\sum_{j=1}^{\infty} \sum_{p=-j}^{j-1} \frac{1}{j^{2}}=\sum_{j=1}^{\infty} \frac{2 j}{j^{2}}=\sum_{j=1}^{\infty} \frac{2}{j}
$$


and similarly

$$
\sum_{k=0}^{\infty}\left|\Delta b_{k}\right|=\sum_{k=1}^{\infty} \frac{2}{k}
$$

Therefore, as $\Delta a_{j}=0$ for $j \leq-1$, and $\Delta b_{k}=0$ for $k \leq-1$, we have

$$
\begin{aligned}
\sum_{j=-\infty}^{\infty} \sum_{k=-\infty}^{\infty}\left|\Delta_{11} c(j, k)\right| & =\sum_{j=-\infty}^{\infty} \sum_{k=-\infty}^{\infty}\left|\left(\Delta a_{j}\right)\left(\Delta b_{k}\right)\right|=\sum_{j=0}^{\infty} \sum_{k=0}^{\infty}\left|\left(\Delta a_{j}\right)\left(\Delta b_{k}\right)\right| \\
& =\left(\sum_{j=0}^{\infty}\left|\Delta a_{j}\right|\right)\left(\sum_{k=0}^{\infty}\left|\Delta b_{k}\right|\right) \\
& =\left(\sum_{j=1}^{\infty} \frac{2}{j}\right)\left(\sum_{k=1}^{\infty} \frac{2}{k}\right)=\infty,
\end{aligned}
$$

which proves that $\{c(j, k)\}$ is not of bounded variation.

But, for $-j \leq p \leq-2$,

$$
\Delta^{2} a_{j^{2}+p}=\Delta a_{j^{2}+p}-\Delta a_{j^{2}+p+1}=\left(\frac{-1}{j^{2}}\right)-\left(\frac{-1}{j^{2}}\right)=0,
$$

for $0 \leq p \leq j-1$,

$$
\Delta^{2} a_{j^{2}+p}=\Delta a_{j^{2}+p}-\Delta a_{j^{2}+p+1}=\left(\frac{1}{j^{2}}\right)-\left(\frac{1}{j^{2}}\right)=0,
$$

and, for $p=-1$ we have

$$
\Delta^{2} a_{j^{2}-1}=\Delta a_{j^{2}-1}-\Delta a_{j^{2}}=\left(\frac{-1}{j^{2}}\right)-\left(\frac{1}{j^{2}}\right)=\frac{-2}{j^{2}} .
$$

Similarly, $\Delta^{2} b_{k^{2}+q}=0$, for $-k \leq q \leq-2$, for $0 \leq q \leq k-1$, and $\Delta^{2} b_{k^{2}-1}=-2 / k^{2}$. Hence, as $\Delta^{2} a_{j}=0$, for $j \leq-2$, and $\Delta^{2} b_{k}=0$, for $k \leq-2$, we have

$$
\begin{aligned}
& \sum_{j=-\infty}^{\infty} \sum_{k=-\infty}^{\infty}\left|\Delta_{22} c(j, k)\right|=\sum_{j=-\infty}^{\infty} \sum_{k=-\infty}^{\infty}\left|\left(\Delta^{2} a_{j}\right)\left(\Delta^{2} b_{k}\right)\right|=\sum_{j=-1}^{\infty} \sum_{k=-1}^{\infty}\left|\left(\Delta^{2} a_{j}\right)\left(\Delta^{2} b_{k}\right)\right| \\
= & \left|\left(\Delta^{2} a_{-1}\right)\left(\Delta^{2} b_{-1}\right)\right|+\left|\left(\Delta^{2} a_{-1}\right)\left(\Delta^{2} b_{0}\right)\right|+\left|\left(\Delta^{2} a_{0}\right)\left(\Delta^{2} b_{-1}\right)\right| \\
& +\left(\sum_{j=0}^{\infty}\left|\Delta^{2} a_{j}\right|\right)\left(\sum_{k=0}^{\infty}\left|\Delta^{2} b_{k}\right|\right) \\
= & |1 \cdot 1|+|1 \cdot(-2)|+|(-2) \cdot 1|+\left(\sum_{j=1}^{\infty} \sum_{p=-j}^{j-1}\left|\Delta^{2} a_{j^{2}+p}\right|\right)\left(\sum_{k=1}^{\infty} \sum_{q=-k}^{k-1}\left|\Delta^{2} b_{k^{2}+q}\right|\right) \\
= & 5+\left(\sum_{j=1}^{\infty} \frac{2}{j^{2}}\right)\left(\sum_{k=1}^{\infty} \frac{2}{k^{2}}\right)<\infty .
\end{aligned}
$$


So, $\{c(j, k)\}$ satisfies $(3.3)$ for $p=2$. Now, for each fixed $k_{0} \in \mathbb{Z}$, we have

$$
\sum_{j=0}^{\infty}\left|\Delta_{20} c\left(j, k_{0}\right)\right|=\sum_{j=0}^{\infty}\left|\Delta^{2} a_{j} b_{k_{0}}\right|=\left(\sum_{j=0}^{\infty}\left|\Delta^{2} a_{j}\right|\right)\left|b_{k_{0}}\right|=\left(\sum_{j=1}^{\infty} \frac{2}{j^{2}}\right)\left|b_{k_{0}}\right|<\infty
$$

and in view of $\left|b_{k_{0}}\right| \rightarrow 0$ as $\left|k_{0}\right| \rightarrow \infty$, it follows that $\{c(j, k)\}$ satisfies (3.4) for $p=2$. Similarly, $\{c(j, k)\}$ satisfies (3.5) also for $p=2$. Thus, $\{c(j, k)\}$ is of bounded variation of order 2 .

Example 3.2. Consider the sequences $\left\{a_{j}\right\}$ and $\left\{b_{k}\right\}$ defined in Example 3.1. Let $\left\{a_{j}^{\prime}\right\}$ and $\left\{b_{k}^{\prime}\right\}$ be sequences defined on $\mathbb{Z}$ such that $a_{0}^{\prime}=0, b_{0}^{\prime}=0$, and $\Delta a_{j}^{\prime}=a_{j}$ and $\Delta b_{k}^{\prime}=b_{k}$, for $j, k \in \mathbb{Z}$. These sequences $\left\{a_{j}^{\prime}\right\}$ and $\left\{b_{k}^{\prime}\right\}$ can be constructed as follows. By our definition, we have $a_{i}=0$, if $i \leq-1$, and the elements $a_{0}, a_{1}, a_{2}, \ldots$ are:

$$
\frac{0}{1}, \frac{1}{1} ; \frac{0}{4}, \frac{1}{4}, \frac{2}{4}, \frac{1}{4} ; \frac{0}{9}, \frac{1}{9}, \frac{2}{9}, \frac{3}{9}, \frac{2}{9}, \frac{1}{9}, \ldots
$$

In view of $0=a_{0}=\Delta a_{0}^{\prime}=a_{0}^{\prime}-a_{1}^{\prime}$ and $a_{0}^{\prime}=0$, we calculate $a_{1}^{\prime}=0$. Then, from $1=a_{1}=\Delta a_{1}^{\prime}=a_{1}^{\prime}-a_{2}^{\prime}$ and from $a_{1}^{\prime}=0$, we calculate $a_{2}^{\prime}=-1$. Similarly, we can calculate all other elements of $\left\{a_{j}^{\prime}\right\}$ and $\left\{b_{k}^{\prime}\right\}$.

Now, we put $c^{\prime}(j, k)=a_{j}^{\prime} b_{k}^{\prime}$, for $j, k \in \mathbb{Z}$. Then, as in Example 3.1, we can easily see that $\left\{c^{\prime}(j, k)\right\}$ is of bounded variation of order 3 but not of bounded variation of order 2 .

Continuing in this way, for each $p \in \mathbb{N}$, we can construct a sequence of bounded variation of order $p+1$ which not of bounded variation of order $p$.

This shows that $(\mathfrak{B V})^{p}$ is a proper subset of $(\mathfrak{B V})^{p+1}$ for each $p \in \mathbb{N}$.

Acknowledgements. This research was completed while the first author was under a visit of Bolyai Institute, University of Szeged, Szeged, Hungary, under the Hungarian State Scholarship Grant Award during the academic year 2016-2017 between May 16, 2017 to June 15, 2017. For this visit, the travel expense was covered by the Financial Assistance under U.G.C. "Travel Grant for the year 2017-2018", The Maharaja Sayajirao University of Baroda, Vadodara, Gujarat, India.

\section{REFERENCES}

[1] C. P. Chen and C. T. Wu, Double Walsh series with coefficients of bounded variation of higher order, Trans. Amer. Math. Soc. 350 (1) (1998), 395-417.

[2] J. W. Garrett, C. S. Rees and Č. V. Stanojević, $L^{1}$-convergence of Fourier series with coefficients of bounded variation, Proc. Amer. Math. Soc. 80(3) (1980), 423-430.

[3] K. Kaur, S. S. Bhatia and B. Ram, Double trigonometric series with coefficients of bounded variation of higher order, Tamkang J. Math. 35(3) (2004), 267-280.

[4] F. Móricz, Convergence and integrability of double trigonometric series with coefficients of bounded variation, Proc. Amer. Math. Soc. 102(3) (1988), 633-640. 
${ }^{1}$ Department of Mathematics,

FACUlTy OF SCIENCE,

The Maharaja Sayajirao University of Baroda,

VADODARA - 390002 ,

GuJARAT, INDIA

Email address: bhikhu_ghodadra@yahoo.com

${ }^{2}$ Bolyai Institute,

UNIVERSITY OF SZEGED,

ARAdi VÉRTANÚK TERE 1 ,

SzEGED 6720,

HUNGARY

Email address: fulopv@math.u-szeged.hu 